\title{
Differential rotation of stars with multiple transiting planets
}

\author{
Yuri Netto and Adriana Valio \\ Center for Radio Astronomy and Astrophysics (CRAAM) Mackenzie Presbyterian University, \\ Sao Paulo, Brazil \\ email: dirceuyuri@hotmail.com
}

\begin{abstract}
If a star hosts a planet in an orbit such that it eclipses the star periodically, can be estimated the rotation profile of this star. If planets in multiplanetary system occult different stellar areas, spots in more than one latitude of the stellar disc can be detected. The monitored study of theses starspots in different latitudes allow us to infer the rotation profile of the star. We use the model described in Silva (2003) to characterize the starspots of Kepler-210, an active star with two planets. Kepler-210 is a late K star with an estimated age of $350 \pm 50$ Myrs, average rotation period of 12.33 days, mass of $0.63 \mathrm{M}_{\odot}$ and radius of $0.69 \mathrm{R}_{\odot}$. The planets that eclipses this star have radii of $0.0498 \mathrm{R}_{s}$ and $0.0635 \mathrm{R}_{s}$ with orbital periods of $2.4532 \pm 0.0007$ days and $7.9725 \pm 0.0014$ days, respectively, where $R_{s}$ is the star radius.
\end{abstract}

Keywords. stars: activity, planetary systems, spots

\section{Introduction}

If a star is eclipsed by an orbiting planet periodically, it is possible to estimate its stellar rotation profile. Small variations in the bottom of the light curve transits may be seen during the transit of the planet. These variations can be interpreted as the planet occulting a spot on the photosphere of the star. Based on these signatures, Silva (2003) developed a method that allows the detection of spots as small as 0.2 planetary radii. Through this method it is also possible to infer properties of individual starspots occulted by the planet, such as size, intensity, and position. This technique was already applied to HD 209458 (Silva 2003), to CoRoT-2 (Silva-Valio \& Lanza 2011), and to Kepler-17 (Valio et al. 2016). By monitoring spots on later transits or considering the fortuitous geometry of Kepler-63, which presents a planet orbiting in an almost polar orbit, the stellar differential rotation was estimated, since it eclipses different stellar latitudes (Netto \& Valio 2016).

In the case of a multiplanetary system, it is possible to infer the stellar rotation profile if the planets occult starspots in different regions of the star, transiting in more than one latitude of the stellar disc. The monitored study of theses starspots at different latitudes allow us to infer the rotation profile of the star.

\section{Kepler-210}

Kepler-210 is an active star, late K star with an estimated age of $350 \pm 50$ Myrs. With a radius of $0.69 \mathrm{R}_{\odot}$, mass of $0.63 \mathrm{M}_{\odot}$ and an average rotation period of 12.33 days, it hosts two transiting Neptune-like planets (Ioannidis et al. 2014).

We have applied the model presented by Silva (2003) to simulate the planetary transits in front of the star Kepler-210. This model assumes a synthesized 2D image of the star with limb-darkening, whereas the planet has a circular orbit. 


\begin{tabular}{cc}
\hline \multicolumn{2}{c}{ Star } \\
\hline $\mathrm{M}\left(\mathrm{M}_{\odot}\right)$ & 0.63 \\
$\mathrm{R}\left(\mathrm{R}_{\odot}\right)$ & 0.69 \\
$\mathrm{P}_{\text {rot }}($ day) & $12.33 \pm 0.15$ \\
Age (Myr) & $350 \pm 50$ \\
$\mathrm{~T}_{\text {eff }}(\mathrm{K})$ & 4300 \\
Linear Limb-Darkening coeff., $\mathrm{u}_{1}$ & $0.8536^{*}$ \\
Quadratic Limb-Darkening coeff. $\mathrm{u}_{2}$ & $0.1990^{*}$ \\
\hline
\end{tabular}

\begin{tabular}{ccc}
\hline \multicolumn{3}{c}{ Planets } \\
& Kepler-210b & Kepler-210c \\
\hline $\operatorname{Rp}\left(\mathrm{R}_{s}\right)$ & $0.0490^{*}$ & $0.0590^{*}$ \\
$\mathrm{a}\left(\mathrm{R}_{s}\right)$ & $7.8706^{*}$ & $22.449^{*}$ \\
$\mathrm{i}(\mathrm{deg})$ & $83.61^{*}$ & $88.87^{*}$ \\
\hline
\end{tabular}

Table 1. Parameters of Kepler-210 system. Those marked by an asterisk are the fitted values found in this work.
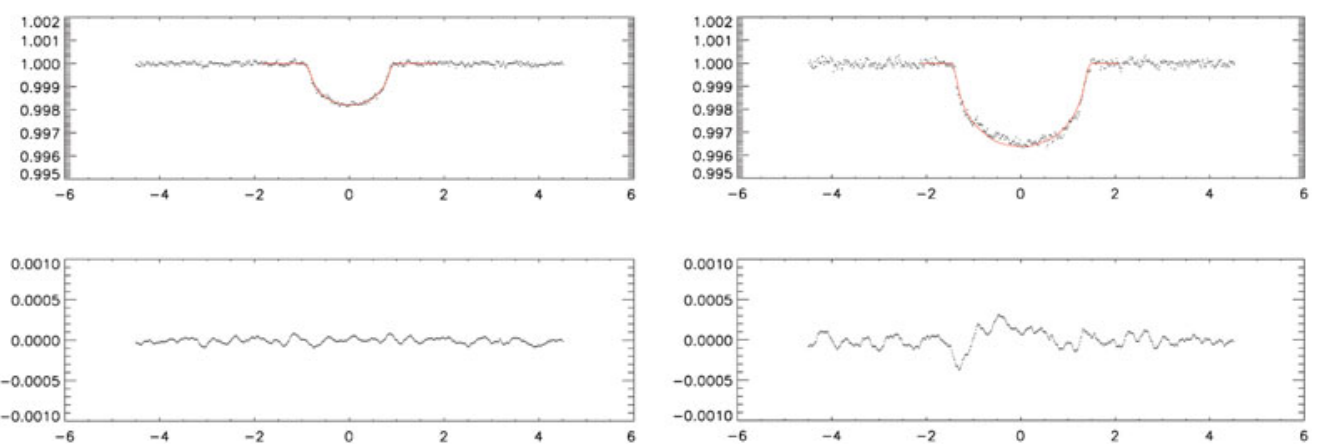

Figure 1. Top: Binned light curve with the fit (red curve) for the transits of planet Kepler-210b (left) and Kepler-210c (right). Bottom: The residuals of the subtraction of the light curve from the modelled spotless transit light curve.

To obtain the spotless light curve for the planet Kepler-210b, we used 302 transits. The data from these transits were binned into a single transit and fitted by a least $\chi$ square minimisation routine (AMOEBA) using the model detailed in Silva (2003) but without spots. The five parameter that were fitted are planet radius, semi-major axis, inclination angle for the planets and limb darkening coefficients for the star. Different values resulted from this fit with respect to the values reported by Ioannidis et al. (2014). The same procedure was applied to the 86 transits of the larger planet, Kepler-210c. The values we obtained are listed in Table 1, and the resulting fits for planets Kepler-210b and Kepler-210c are shown as the red line in the top panels of the Figure 1. The residuals of the subtraction of this model from the binned light curve are plotted on the bottom panels of Figure 1.

A simulation of the star Kepler-210 and its planets are presented in the Figure 2. To the values fitted in this work, the planets should eclipse the star at the stellar latitudes of $-61^{\circ}$ and $-26^{\circ}$ for planets Kepler-210b and Kepler-210c, respectively.

Unfortunately, no spots signatures in the residuals were found above the detection threshold of 10 times the CDPP (Combined Differential Photometric Precision, Christiansen et al. (2012)). 


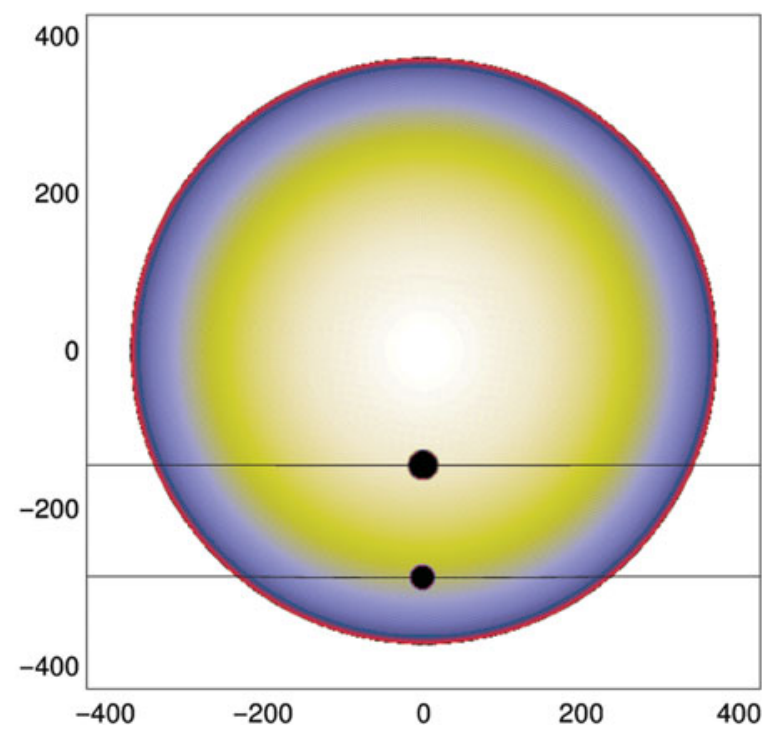

Figure 2. Planetary transit simulation for the two planets orbiting around Kepler-210.

Kepler-210c transits closer to the stellar pole, while Kepler-210b is transiting at mid-latitudes.

\section{Summary and Conclusions}

Using the method described in Silva (2003), we modelled the light curve of the star Kepler-210. A total of 302 transits were used for the first planet, and 86 for the second one. The fitted parameters resulted in an increase in the semi-major axis and in the orbital inclination angle with respect to those reported by Ioannidis et al. (2014). On the other hand, the fits resulted in a decrease in the planet radius of $1.6 \%$ and $7.0 \%$ for the Kepler-210b and Kepler-210c, respectively. Although, Kepler-210 is an active young star, its noise is such that it drowns the signature of any spot in the transit light curve of its planets.

\section{References}

Christiansen, J. L., Jenkins, J. M., Caldwell, D. A., et al. 2012, PASP, 124, 1279

Ioannidis, P., Schmitt, J. H. M. M., Avdellidou, C., von Essen, C., \& Agol, E. 2014, A\&A, 564, A33

Netto, Y.,\& Valio, A. 2016, ApJLett (Submitted)

Silva, A. V. R. 2003, ApJLett, 585, 147

Silva-Valio, A. \& Lanza, A. F. 2011, A\&A, 529, A36

Valio, A., Estrela, R., Netto, Y., Bravo, J. P., \& Medeiros, J. R. 2016, ApJ (Accepted) 